\title{
Imaging features of ultrasound and contrast-enhanced ultrasound in primary hepatic carcinosarcoma: three cases report
}

\author{
Chun-Rui Liu, Shu-Ping Wei, Min Wu, Wen-Tao Kong
}

Department of Ultrasound, Nanjing Drum Tower Hospital, The Affiliated Hospital of Nanjing University Medical School, Nanjing, China

\begin{abstract}
Primary hepatic carcinosarcoma (PHCS) is a very rare malignant tumor with a poor prognosis. Previous studies in the English-language literature were case reports, focused on the clinical aspects, pathology and pathogenesis. Much is unknown about the imaging characteristics of PHCS while ultrasound is the first imaging examination for hepatic disease. Contrastenhanced ultrasound (CEUS) is a proven method that improves the detection and characterization of focal liver lesions. The purpose of the paper is to present the ultrasonographic and other imaging appearance of three cases of PHCS.

Keywords: liver; carcinosarcoma; diagnosis; ultrasound; contrast-enhanced ultrasound (CEUS)
\end{abstract}

\section{Introduction}

The WHO defines carcinosarcoma as a malignant tumor composed of a mixture of carcinomatous and sarcomatous elements. Primary hepatic carcinosarcoma (PHCS) is a very rare malignant tumor which is highly invasive, metastatic and with a poor prognosis [1]. The symptoms and serological tumor markers are nonspecific and the prognosis of PHCS is mainly related to tumor size, differentiation degree, clinical stage and surgical excision range.

Received 29.04.2019 Accepted 24.07.2019

Med Ultrason

2019, Vol. 21, No 4, 487-490

Corresponding author: Wen-Tao Kong, $\mathrm{PhD}$

Department of Ultrasound, Nanjing Drum Tower Hospital, The Affiliated Hospital of Nanjing University Medical School,

321 Zhongshan Road, 210008, Nanjing, China

E-mail: breezewen@163.com
Previous published papers mostly reviewed the pathogenesis and clinical characteristics [2-5] or described the imaging features in computed tomography (CT), magnetic resonance imaging (MRI) or ${ }^{18} \mathrm{~F}$-fluorodeoxyglucose positron emission tomography (PET) of PHCS [6-8]. Little is known about ultrasound (US) and contrast-enhanced ultrasound (CEUS) [9]. We summarized three cases of PHCS confirmed by pathology and immunohistochemistry (IHC) after surgery or biopsy. The findings of relevant imaging features and literature were reviewed.

\section{Cases report}

\section{Case 1}

A 59-year-old man was admitted for an asymptomatic hepatic nodule which was incidentally found by US on a routine medical check-up. Carbohydrate antigen 125 (CA125) was in normal range (41.68 U/ml). US, CT and 
PET examinations showed multiple lesions in the right hepatic lobe, right diaphragm and right pleura (fig 1 and 2 ). Right hemihepatectomy and phrenectomy was performed. Pathological diagnosis revealed spindle cells were positive for vimentin (+++), CK8/18 and Ki 67 positive in $80 \%$ of cells. The patient died five months later.

\section{Case 2}

An 85-year-old woman was referred to our hospital because of fever and right upper abdominal pain for one month. The highest temperature was $38.5^{\circ} \mathrm{C}$. She had a history of coronary heart disease, cerebral infarction and hypertension for more than 10 years. The serum alphafetoprotein (AFP) was $25.7 \mathrm{ng} / \mathrm{ml}$. US and MRI findings are shown in fig 3 and 4. Liver abscess was considered. Ultrasound-guided percutaneous liver biopsy was performed. IHC were positive for vimentin $(++)$, S100, CK8/18. The patient died five months later.

\section{Case 3}

A 76-year-old woman presented in our hospital due to abdominal pain and distension for 12 months. Past medical history included hepatic B virus infection and entecavir anti-viral treatment for 5 years. Tumor markers were normal. US diagnosis was likely to be primary hepatic carcinoma (HCC) (fig 5). The patient underwent laparoscopic right posterior lobe hepatectomy. IHC was positive for vimentin (++), CK7/20, CD 10 and CK19. Patient was alive during preparation of the manuscript.

\section{Discussions}

On US firstly, PHCS appears to be solitary and very large, such as Cases 1 and 2, occupying more than one segment and even a whole lobe. The tumor was heterogeneous hypoechoic or mix-echoic mass lesions with irregular and obscure margin. However, when a tumor was
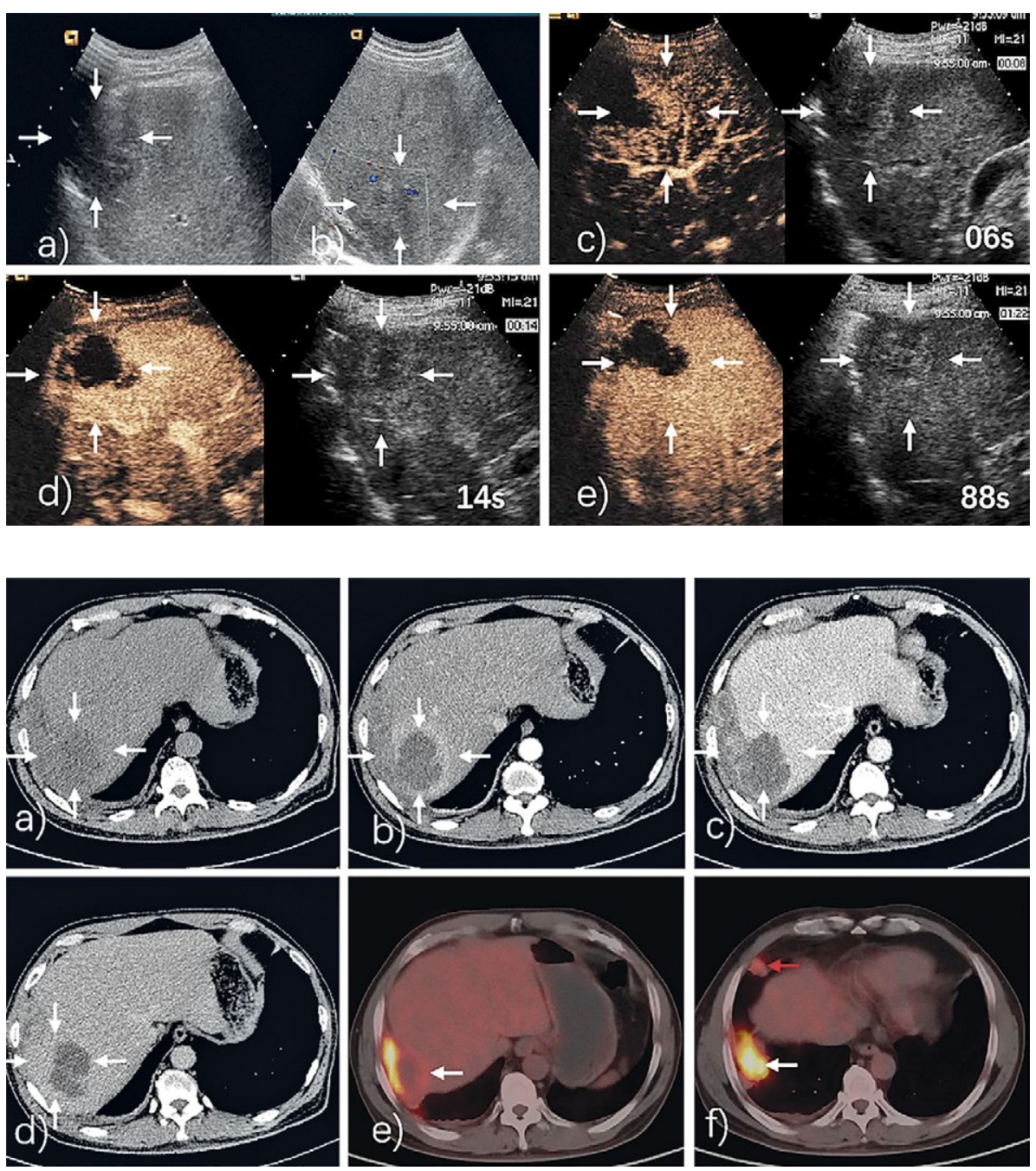

Fig 1. Case 1: Ultrasound illustrated an ill-defined heterogeneous mass sized $8 \mathrm{~cm}$ at right lobe (a). There was punctiform CDFI signals detected at different ultrasonic cross-sections (b). CEUS images obtained at 6,14 and 88 seconds showed early arterial hyperenhancement (c) with necrotic areas (d) and marked wash-out (e).

Fig 2. Case 1: CT showed an obscure-defined low-density mass in the right lobe (a). Contrastenhanced CT showed the tumor enhancement during the arterial phases (b), portal venous(c) and delayed washout in the latter phases (d). PET-CT image showed a low-density liver mass with a maximum standardized uptake value (SUVmax) of 7.4 (white arrowhead) (e) and right diaphragm nodule with SUVmax of 4.0 (red arrowhead). 
small, like Case 3, it appeared a homogenous hypoechoic nodule with regular and distinct margin. Secondly, the tumor located close to the liver capsule presents extrahepatic involvement due to the fact that sarcomatous cell proliferation is very rapid. Case 1 was associated with a diaphragmatic tumor. Thirdly, calcification is rare in HCC, but when sarcomatous components demonstrate typically specialized differentiation such as osteoid,
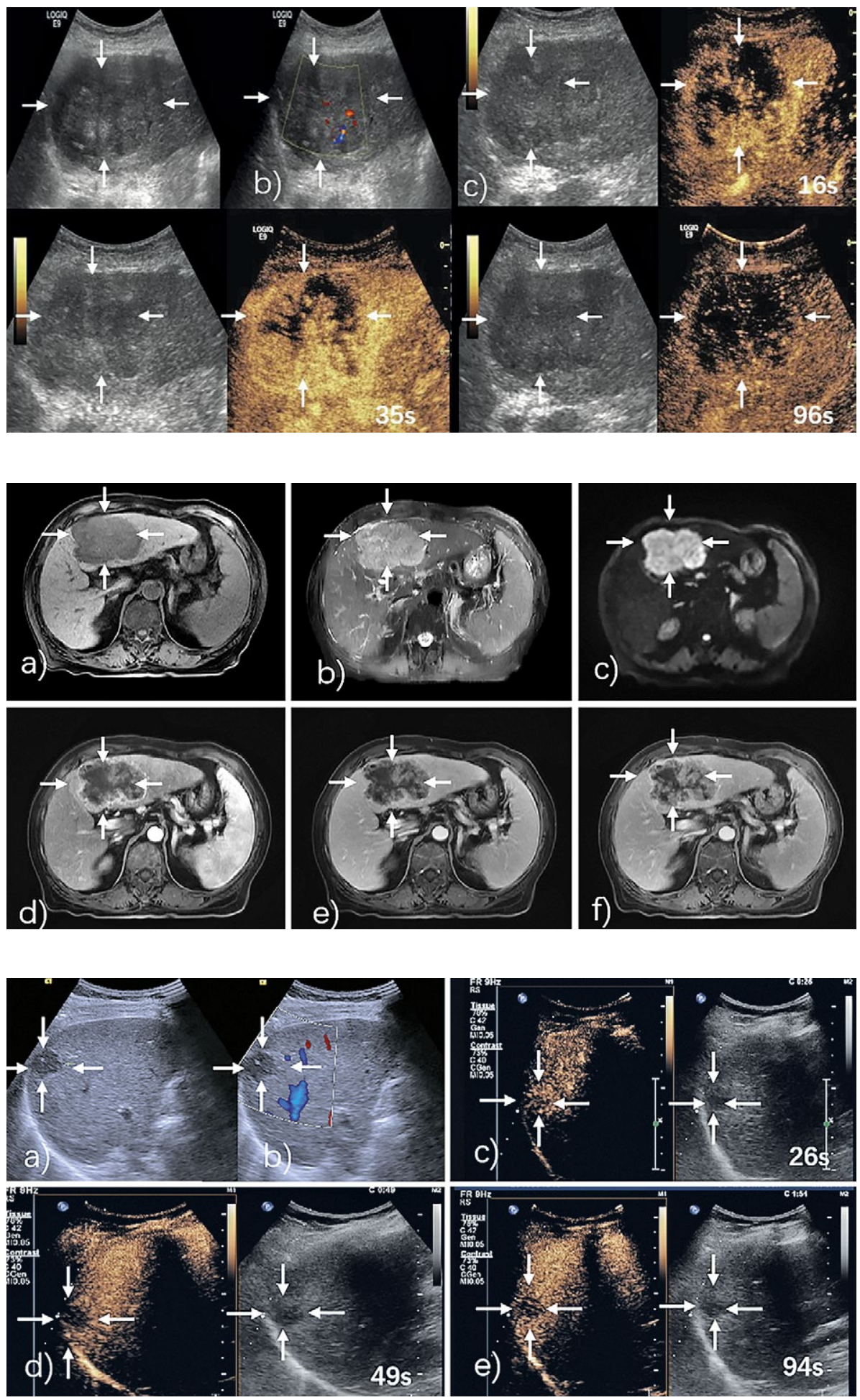

Fig 3. Case 2: Ultrasound showed a heterogeneous hypoechoic mass sized $7.9 \mathrm{~cm}$ with poorly defined margin (a) and punctiform blood signals (b). CEUS images obtained at 16, 35 and 96 seconds showed heterogeneous arterial hyperenhancement with necrotic areas (c), peripheral irregular rim-like enhancement (d) and marked washout comparison with adjacent-liver tissue (e).

Fig 4. Case 2: T1-weighted revealed a heterogeneous hypointense mass in the left lobe (a). Fat suppressed T2-weighted showed a heterogeneous hyperintense mass (b). Diffusion-weighted imaging showed higher intensity than normal liver parenchyma (c). Contrast-enhanced MR revealed heterogeneous significant enhancement in the arterial phase (d), fading fast washout in the portal venous (e) and delay phase (f).

Fig 5. Case 3: Ultrasound showed a hypoechoic solid nodule sized $2.5 \mathrm{~cm}$ and a highly echogenic within the mass (a). There was no blood flow on CDFI (b). CEUS images obtained at 26, 49 and 94 seconds showed diffuse isoenhancement (c) with a blurring boundary (d) and a marked washout comparison with adjacentliver tissue (e). 
rocky hyper echo may be seen within the mass [10]. We found this in Case 3. Hyperechoic calcification may be the classic sign of PHCS. Our cases were hypovascular tumors (punctiform or inconspicuous blood flow signals).

On CEUS, PHCS displayed peripheral nodular enhancement in the arterial phase and hypo-enhancing in the later phase. The tumor also displayed a large internal non-enhancing portion, because the interior of PHCS often exhibits hemorrhage and necrosis [9]. The added value of CEUS is related to the fact that the technique can demonstrate the boundary and internal components of PHCS and assist in differential with HCC, liver abscess or intrahepatic cholangiocarcinoma (ICC). HCC shows irregular hypervascularization while PHCS shows peripheral nodular enhancement in the arterial phase. Although liver abscess shows rim enhancement, the internal of abscess shows no enhancement areas or honeycomb appearance. It is very difficult to differentiate ICC from PHCS. ICC also shows peripheral enhancement and central hypo-enhancing $[11,12]$.

Plain CT of PHCS revealed an irregularly demarcated hypodensity intrahepatic mass [10]. On MR, the tumor appeared as a heterogeneous hypointense on T1weighted and heterogeneous hyperintense lesion on T2weighted [7]. The enhancement of CT and MR is like that of US. PET may be a useful diagnostic tool for PHCS because the high fluorodeoxyglucose uptake of sarcomatous element seems to be related to its progression or aggressiveness [8].

In conclusion, PHCS is an extremely rare and aggressive tumor. Imaging modalities are useful for detecting the differential diagnosis of tumors. US and CEUS are excellent tools for the characterization of liver lesions; more cases remain to be identified and summarized.

\section{Acknowledgements}

This study was funded by the National Natural Science Foundation of China (81671701) and the Nanjing Medical Science and technique Development Foundation (QXR17011)

\section{References}

1. Kurita D, Mokuno Y, Matsubara H, et al. Primary hepatic carcinosarcoma with multimodal treatment. Nagoya J Med Sci 2018;80:423-429.

2. Lu J, Zhang J, Xiong XZ, et al. Primary hepatic sarcomatoid carcinoma: clinical features and prognosis of 28 resected cases. J Cancer Res Clin Oncol 2014;140:10271035.

3. Lin YS, Wang TY, Lin JC, et al. Hepatic carcinosarcoma: clinicopathologic features and a review of the literature. Ann Hepatol 2013;12:495-500.

4. Lao XM, Chen DY, Zhang YQ, et al. Primary carcinosarcoma of the liver: clinicopathologic features of 5 cases and a review of the literature. Am J Surg Pathol 2007;31:817826.

5. Mitra S, Gupta S, Dahiya D, Saikia UN. A Rare Case of Primary Sarcomatous Hepatocellular Carcinoma Without Previous Anticancer Therapy. J Clin Exp Hepatol 2017;7:378384.

6. Li J, Liang P, Zhang D, et al. Primary carcinosarcoma of the liver: imaging features and clinical findings in six cases and a review of the literature. Cancer Imaging 2018;18:7.

7. Yasutake T, Kiryu S, Akai H, et al. MR imaging of carcinosarcoma of the liver using Gd-EOB-DTPA. Magn Reson Med Sci 2014;13:117-121.

8. Ijichi H, Shirabe K, Taketomi A, et al. Clinical usefulness of (18) F-fluorodeoxyglucose positron emission tomography/computed tomography for patients with primary liver cancer with special reference to rare histological types, hepatocellular carcinoma with sarcomatous change and combined hepatocellular and cholangiocarcinoma. Hepatol Res 2013;43:481-487.

9. Liu LP, Yu XL, Liang P, Dong BW. Characterization of primary hepatic carcinosarcoma by contrast-enhanced ultrasonography: a case report. World J Gastroenterol 2014;20:1630-1634.

10. Kwon JH, Kang YN, Kang KJ. Carcinosarcoma of the liver: a case report. Korean J Radiol 2007;8:343-347.

11. Ferraioli G, Meloni MF. Contrast-enhanced ultrasonography of the liver using SonoVue. Ultrasonography 2018;37:25-35.

12. Popescu A, Sporea I, Sirli R, et al. Does Contrast Enhanced Ultrasound improve the management of liver abscesses? A single centre experience. Med Ultrason 2015;17:451-455. 